\title{
A THEORY OF NONLINEAR WAVE LOADING ON OFFSHORE STRUCTURES
}

\author{
LOKENATH DEBNATH \\ Mathematical Institute \\ University of Oxford \\ Oxford, England.
}

And

\section{MATIUR RAHMAN}

Hydraulics Laboratory

Division of Mechanical Engineering

National Research Council of Canada

Ottawa, Canada.

(Received in May 1, 1980 and in revised form June 25, 1980)

ABSTRACT. A theoretical study is made of the nonlinear wave loading on offshore structures using the diffraction theory of hydrodynamics. A nonlinear modification of the classical Morison equation, $D \equiv F_{\ell}+F_{D}$ for estimating wave forces on offshore structures is suggested in this paper. The modified equation is found in the form $D \equiv F_{\ell}+F_{n \ell}+F_{D}$, where $F_{n \ell} \equiv F_{d}+F_{w}+F_{q}$ is the nonlinear contribution made up of the dynamic, waterline, and the quadratic forces associated with the irrotational-flow part of the wave loading on structures. The study has then been applied to calculate the linear and the nonlinear wave loadings on a large vertical cylinder partially immersed in an ocean of arbitrary uniform depth. All the linear and nonlinear forces exerting on the cylinder are determined explicitly. "A comparison is made between these two kinds of forces. Special attention is given to the nonlinear wave loadings on the cylinder. It is shown that all nonlinear effects come from the interaction between the body's responses to the oncoming wave's fluctuating velocity and its fluctuating extension. It is found 
that the nonlinear effects are dominated by the sum of the dynamic and waterline forces. The nonlinear correction to Morison's equation increases with increasing $\mathrm{kb}$ where $\mathrm{b}$ is the characteristic dimension of the body and $\mathrm{k}$ is the wave number. This prediction is shown to be contrary to that of the linear diffraction theory which predicted that the Morison coefficient decreases with increasing $\mathrm{kb}$. Several interesting results and limiting cases are discussed in some detail.

KEY WORDS AND PHRASES. Nonlinear waves, wave Forces, Morison equation, wave loading on Structures, Hydrodynamic Diffraction 1980 MATHEMATICS SUBJECT CLASSIFICATION CODES. 76B15, $76 B 20$.

\section{INTRODUCTION.}

One of the most common problems in hydrodynamics deals with waves as well as wave loading estimation for offshore structures. This problem is of considerable mathematical and engineering interest due to abundant applications to marine hydrodynamigs. In order to indicate our motivation and interest in the problem, it is necessary to review some of the relevant works on the subject.

Based upon the classical works on linear diffraction theory for water waves by Havelock [1] and for sound waves by Morse [2], MacCamy and Fuchs [3] developed a linear diffraction theory for wave forces on a large cylindrical pile immersed in oceans. This study was found to have limited applications because the nature of water waves is inherently nonlinear.

In recent papers [4-5], Chakraborty has made an interesting extension of MacCamy and Fuch's work, and obtained analytical solutions of the second order wave forces on a vertical cylinder based upon the Stokes fifth-order wave theory. It is unfortunate that his scattered potential fails to satisfy the nonlinear free surface boundary condition and the radiation condition at infinity. In order to eliminate these drawbacks of Chakraborty's theory, Raman and Venkatanarasaiah [6] have presented a nonlinear diffraction theory due to the Stokes second-order waves for estimating the wave forces on a vertical cylinder. Although their work has provided some interesting theoretical and experimental results, the method of solution is extremely complicated. 
Historically, the wave loading estimation for offshore structures has been based upon the work of Morison et al [7]. Morison's equation is generally used for calculating wave forces on solid structures in oceans. This equation expresses the total drag $D$ as a sum of the inertial force, $\rho C_{M} V \dot{U}$ associated with the irrotational flow component, and the drag force, $\frac{1}{2} \rho C_{D} A U^{2}$ related to the vortex-flow component of the fluid flows. Mathematically, the Morison equation is

$$
D=\rho C_{M} V \dot{U}+\frac{1}{2} \rho C_{D} A U^{2}
$$

where $\rho$ is the fluid density, $C_{M}=\left(1+\frac{M}{\rho V}\right)$ is the Morison (or inertia1) coefficient, $M_{a}$ is the added mass, $V$ is the volumetric displacement of the body, $U$ is the fluctuating fluid velocity along the horizontal direction, $A$ is the projected frontal area of the wake vortex, and $C_{D}$ is the drag coefficient. It is assumed that the inertial and drag forces acting on a fixed solid object in an unsteady fluid flow are independent in the sense that there is no interaction between them.

There are two characteristic features of the Morison equation. One deals with the nature of the inertial force which is linear in velocity $U$. The other indicates that the drag force is nonlinaer in velocity $U$. It is generally believed that all nonlinear effects in experimental data are associated with drag forces. However, for real solid structures in real ocean waves, there is a significant nonlinear force associated with the irrotational component of the fluid flow because of the large amplitude of ocean waves. These waves are of special interest in wave loading estimation. It seems necessary to incorporate all significant nonlinear effects associated with the nonlinear free surface boundary condition in the irrotational flow part of the wave loading on structures.

Several studies have shown that the mathematical form of the Morison equation is satisfactory. However, certain difficulties in using it in the design and construction of offshore structures have been reported in the literature. These are concerned with the drag force which has relatively large scale effects. The shortage of reliable full-scale drag data in wave motions is also another problem. 
There is another important question whether a linear theory of the irrotationalflow response is appropriate at all to water wave motions with a free surface. Despite these difficulties, the use of the Morison equation has extensively been documented in the literature through plentiful data for determining the coefficients $C_{M}$ and $C_{D}$.

Recently, Hogben et a1 [8] and Lighthil1 [9] have published comprehensive review articles on the recent developments in the subject with a critical evaluation of both the methods and the results concerning the waves and hydrodynamic loading on offshore structures. Lighthill has also suggested that the Morison equation needs to be amended by the incorporation of significant nonlinear terms associated with free-surface effects on the irrotational-flow part of the wave loading. He then discussed a general theory and results with many interesting comments on the second-order wave forces on a large vertical cylinder immersed in deep oceans.

Motivated by the above discussion, especially on the nonlinear irrotationalflow response theory, we became interested in the study of nonlinear effects on wave loading estimation in order to examine the significance of these effects and to incorporate them in the Morison equation.

This paper is concerned with the study of the nonlinear wave loading on offshore structures using the diffraction theory of hydrodynamics. A nonlinear modification of the Morison equation for estimating wave forces on offshore structures is presented in this paper. It is shown that this modification is made up of the dynamic, waterline, and the quadratic wave forces associated with the irrotational-flow part of the wave loading on structures. All linear and nonlinear wave loadings on a large vertical cylinder immersed partially in an ocean of arbitrary uniform depth are calculated explicitly. A comparison is made among the various nonlinear forces, and between the linear and nonlinear wave forces in order to determine their relative importance. It is shown that results of Lighthill for the case of deep oceans can be recovered as limiting cases of this analysis. 


\section{BASIC EQUATIONS FOR WATER WAVES.}

We consider a large rigid vertical cylinder of radius $b$ which is acted upon by a train of two-dimensional, periodic, progressive waves propagating on the surface of a fluid of arbitrary uniform depth $h$. We assume that the fluid is inviscid, incompressible, and that the motion is irrotational in the region bounded by the free surface, rigid bottom boundary and the surface of the cylinder.

In this axisymmetric configuration with symmetry about the z-axis vertical positive upward, the velocity potential $\phi(r, \theta, z ; t)$ and the free surface elevation $\eta(r, \theta ; t)$ are governed by the following equations and boundary conditions:

$$
\nabla^{2} \phi \equiv \frac{\partial^{2} \phi}{\partial r^{2}}+\frac{1}{r} \frac{\partial \phi}{\partial r}+\frac{1}{r^{2}} \frac{\partial^{2} \phi}{\partial \theta^{2}}+\frac{\partial^{2} \phi}{\partial z^{2}}=0
$$

where $\mathrm{b} \leq \mathrm{r}<\infty,-\mathrm{h} \leq \mathrm{z} \leq \mathrm{n}$ and $-\pi \leq \theta \leq \pi$.

The dynamic condition at the free surface is

$$
\frac{\partial \theta}{\partial t}+g \eta+\frac{1}{2}(\nabla \phi)^{2}=0 \quad \text { on } z=n, r \geq b
$$

where $g$ is the acceleration due to gravity and the velocity components are $\left(\mathbf{u}_{\mathbf{r}}, \mathrm{u}_{\theta}, \mathrm{u}_{\mathbf{z}}\right) \equiv \nabla \phi=\left(\frac{\partial \phi}{\partial \mathbf{r}}, \frac{1}{\mathbf{r}} \frac{\partial \phi}{\partial \theta}, \frac{\partial \phi}{\partial \mathbf{z}}\right)$.

The kinematic condition at the free surface is

$\frac{\partial \eta}{\partial t}-\frac{\partial \phi}{\partial z}+\left(\frac{\partial \phi}{\partial r}\right) \quad\left(\frac{\partial \eta}{\partial r}\right)+\frac{1}{r^{2}}\left(\frac{\partial \phi}{\partial \theta}\right) \quad\left(\frac{\partial \eta}{\partial \theta}\right)=0 \quad$ on $z=n, r \geq b$

The boundary condition at the rigid bottom is

$$
\frac{\partial \phi}{\partial z}=0 \quad \text { on } z=-h
$$

The radiation condition at infinity is

$$
\lim _{k r \rightarrow \infty}\left[(k r)^{\frac{1}{2}} \quad\left(\frac{\partial}{\partial r} \pm i k\right) \phi_{s}\right]=0
$$

where $k=\frac{2 \pi}{\lambda}$ is the wave number of scattered wave, $\phi=\phi_{l}+\phi_{s}$ is the total velocity potential, $\phi_{\ell}$ and $\phi_{s}$ represent the incident and the scattered wave potentials respectively. 
3. IRROTATIONAL-FLOW PRESSURE DISTRIBUTIONS.

The irrotational-flow pressure distribution can be obtained from the Bernoulli equation which is

$$
\frac{p}{\rho}+g z+\frac{\partial \phi}{\partial t}+\frac{1}{2}(\nabla \phi)^{2}=0
$$

This equation enables us to define three pressure distributions:

(i) The hydrostatic pressure distribution which has the value

$$
\mathrm{p}_{\mathrm{o}}-\rho \mathrm{gz} \text {, }
$$

at a height $z$ above the level $z=0$ where the atmospheric pressure is $p_{0}$.

(ii) The dynamic pressure distribution which has the well known Bernoulli value

$$
\frac{1}{2} \rho\left(u^{2}-q^{2}\right) \text {, }
$$

at a point where the fluid speed is $q$.

(iii) The transient pressure distribution which has the value

$$
-\rho \frac{\partial \phi}{\partial t}
$$

There is a certain resultant force with which each of these pressure components acts on a solid body in a stream of homogeneous liquid of variable speed $U$.

The total derivative of (3.1) with respect to time $t$ has the form

$$
\frac{\mathrm{D}}{\mathrm{Dt}}\left(\frac{\mathrm{p}}{\rho}\right)+\mathrm{g} \frac{\mathrm{Dz}}{\mathrm{Dt}}+\frac{\mathrm{D}}{\mathrm{Dt}}\left(\frac{\partial \phi}{\partial \mathrm{t}}\right)+\frac{1}{2} \frac{\mathrm{D}}{\mathrm{Dt}}(\nabla \phi)^{2}=0,
$$

which can be written as

$$
\frac{\mathrm{D}}{\mathrm{Dt}}\left(\frac{\mathrm{p}}{\rho}\right)+\left(\frac{\partial^{2} \phi}{\partial \mathrm{t}^{2}}+\mathrm{g} \frac{\partial \phi}{\partial z}\right)+\frac{\partial}{\partial t}(\nabla \phi)^{2}+\frac{1}{2} \underline{q} \cdot \nabla(\nabla \phi)^{2}=0,
$$

where $q$ is the velocity vector.

Equation (3.6) has been evaluated at $z=n$ to obtain

$$
\frac{\mathrm{D}}{\mathrm{Dt}}\left(\frac{\mathrm{p}_{\mathrm{o}}}{\rho}\right)+\left(\frac{\partial^{2} \phi}{\partial \mathrm{t}^{2}}+\mathrm{g} \frac{\partial \phi}{\partial t}\right)_{z=n}+\left[\frac{\partial}{\partial t}(\nabla \phi)^{2}\right]_{z=\eta}+\frac{1}{2}\left[q \cdot \nabla(\nabla \phi)^{2}\right]_{z=n}=0 .
$$

If the atmospheric pressure is constant, the first term in (3.7) vanishes so that (3.7) reduces to

$$
\frac{\partial^{2} \phi}{\partial t^{2}}+g\left(\frac{\partial \phi}{\partial z}\right)+\frac{\partial}{\partial t}(\nabla \phi)^{2}+\frac{1}{2}\left[\underline{q} \cdot \nabla(\nabla \phi)^{2}\right]=0 \text { on } z=n .
$$


Retaining up to second-order term in (3.8), we obtain

$$
\frac{\partial^{2} \phi}{\partial t^{2}}+g \frac{\partial \phi}{\partial z}=-\frac{\partial}{\partial t}(\nabla \phi)^{2}
$$

This is the equation derived by Lighthill [9]. It is noted here that a simple combination of equations (2.2) and (2.3) also gives the result (3.9).

4. PERTURBATION OF SOLUTION AND NONLINEAR WAVE FORCES.

We write the velocity potential, $\phi$ and the free-surface elevation function, $\eta$ in the form

$$
\begin{aligned}
& \phi=\phi_{\ell}+\phi_{q}+\ldots \\
& n=n_{l}+n_{q}+\ldots
\end{aligned}
$$

where $\phi_{\ell}, \eta_{\ell}$ are the ordinary solutions satisfying the linearized free-surface conditions and $\phi_{q}, \eta_{q}$ represent the quadratic correction of the order of the squares of the disturbances (and higher order corrections are neglected). Thus the following Taylor series expansion of $\phi$ about $z=0$ holds good and has the form

$$
\begin{aligned}
\phi(r, \theta, n(r, \theta ; t)) & =\phi(r, \theta, 0)+n\left(\frac{\partial \phi}{\partial z}\right)_{z=0}+\ldots . \\
& =\left(\phi_{\ell}+\phi_{q}+\ldots .\right)+\left(\eta_{\ell}+\eta_{q}+\ldots .\right)\left[\frac{\partial \phi_{\ell}}{\partial z}+\frac{\partial \phi}{\partial z}+\ldots\right]_{z=0}, \ldots,(4.3)
\end{aligned}
$$

Therefore "

$\phi=\phi_{\ell}+\phi_{q}+\eta_{\ell}\left(\frac{\partial \phi_{\ell}}{\partial z}\right)_{z=0}+$ (higher order terms),

where the first term is the first-order term and the second one represents the second-order term.

With a similar expansion by the Taylor series, we obtain

$$
\begin{aligned}
& \frac{\partial \phi}{\partial z}=\frac{\partial \phi_{\ell}}{\partial z}+\left\{\frac{\partial \phi_{q}}{\partial z}+n_{\ell}\left[\frac{\partial}{\partial z}\left(\frac{\partial \phi_{\ell}}{\partial z}\right)\right]_{z=0}\right\}+\ldots \\
& \frac{\partial^{2} \phi}{\partial t^{2}}=\frac{\partial^{2} \phi_{\ell}}{\partial t^{2}}+\left\{\frac{\partial^{2} \phi_{\ell}}{\partial t^{2}}+n_{\ell}\left[\frac{\partial}{\partial z} \frac{\partial^{2} \phi_{\ell}}{\partial t^{2}}\right]_{z=0}\right\}+\ldots \\
& \nabla \phi=\nabla \phi_{\ell}+\left\{\nabla \phi_{q}+n_{\ell}\left[\frac{\partial}{\partial z}\left(\nabla \phi_{\ell}\right)\right]_{z=0}\right\}+\ldots
\end{aligned}
$$


Substituting these results into (3.9), we obtain equations for the linear potential $\phi_{\ell}$ and the quadratic potential $\phi_{q}$ in the form

$$
\begin{gathered}
\frac{\partial^{2} \phi_{l}}{\partial t^{2}}+g \frac{\partial \phi_{l}}{\partial z}=0, \\
\frac{\partial^{2} \phi_{q}}{\partial t^{2}}+g \frac{\partial \phi_{q}}{\partial z}=-\frac{\partial}{\partial t}\left(\nabla \phi_{l}\right)^{2}-\eta_{l}\left[\frac{\partial}{\partial z}\left(\frac{\partial^{2} \phi_{l}}{\partial t^{2}}+g \frac{\partial \phi_{l}}{\partial z}\right)\right]_{z=0},
\end{gathered}
$$

where $n_{l}=-\frac{1}{\mathbf{g}}\left(\frac{\partial \phi_{l}}{\partial t}\right)$.

We would like to make some comments on the equation (4.9) satisfied by the quadratic potential $\phi_{q}$, and the additional wave loading generated by $\phi_{q} \cdot$ For making these comments, it is necessary to recall certain results of the theory of irrotational fluid flows which is extremely useful for the study of both steady and unsteady surface waves in oceans. In these studies when solid boundaries are absent, there is no principal source of generation of vorticity. With the linearized boundary condition at the free surface $z=0$, the problem of determining the irrotational flow field becomes a purely linear problem because the velocity potential satisfies the well known Laplace equation. With such linear system of equations, the irrotational-flow response to oncoming waves of every frequency and direction in the ocean-wave spectrum can explicitly be determined. For example, the sinusoidal waves of amplitude a propagating freely in the $x$-direction in water of uniform depth $h$ have the velocity of potential $\phi$, and the free surface elevation $n$ given by

$$
\begin{aligned}
\phi(x, z ; t) & =a \omega \frac{\cosh k(z+h)}{k \sinh k h} \cos (\omega t-k x), \\
n(x, t) & =a \sin (\omega t-k x),
\end{aligned}
$$

where $\omega$ is the frequency and $k$ is the wave number.

The linearized boundary condition at the free surface $z=0$ gives the well known dispersion relation

$$
\omega^{2}=g k \tanh k h .
$$


In the case of deep water, these equations reduce to simple form

$$
\phi=\frac{a \omega}{k} e^{k z} \cos (\omega t-k x), \omega^{2}=g k .
$$

These simply mean that each water particle moves in a circular path at constant speed which decreases exponentially with depth of the 1iquid.

With the aid of the above results (4.10) - (4.12), we can rewrite the equation (4.9) in the form

$$
\frac{\partial^{2} \phi_{q}}{\partial t^{2}}+g \frac{\partial \phi_{q}}{\partial z}=-\frac{\partial}{\partial t}\left[\left(\nabla \phi_{\ell}\right)^{2}+\frac{1}{2}(\tanh k h-1)\left(k \phi_{\ell}\right)^{2}\right]
$$

In the case of infinitely deep water, $k h \rightarrow \infty$ so that $\tanh k h \rightarrow 1$, this equation takes the even simpler form

$$
\frac{\partial^{2} \phi_{q}}{\partial t^{2}}+g \frac{\partial \phi q}{\partial z}=-\frac{\partial}{\partial t}\left(\nabla \phi_{\ell}\right)^{2} \text {. }
$$

In other words, for the deep water case, the second term on the right hand side of (4.9) or (4.14) disappears, and the quadratic potential $\phi_{q}$ satisfies (4.15) at the free surface with zero boundary conditions everywhere else.

Similarly, for a very shallow water, $k h \rightarrow 0$ so that $\tanh k h \rightarrow k h$, we can also write down the corresponding form of the equation (4.14).

It is also noted that the left hand side of equations (4.14) and (4.15) has no terms in $\phi_{\ell}$. This is because of the fact that the potential $\phi_{\ell}$ satisfies the linearized free-surface condition not only on $z=0$ but everywhere in the fluid including $z=n$. Physically, equation (4.14) implies that $\phi_{q}$ is the solution of the linearized wave motion generated in the presence of the body at rest by the fluctuating free-surface pressure distribution

$$
\rho\left[\left(\nabla \phi_{\ell}\right)^{2}+\frac{1}{2}(\tanh k h-1)\left(k \phi_{\ell}\right)^{2}\right] \text { or } \rho\left(\nabla \phi_{\ell}\right)^{2}
$$

according as the fluid is of finite or infinite depth. The frequency of this fluctuating pressure is twice of that of the oncoming waves.

Making reference to Lighthill [9], the quadratic force $F_{q}$ generated by the second-order potential $\phi_{\mathrm{q}}$ is defined by the integral 


$$
F_{q}=-\int_{z=0} W \rho\left[\left(\nabla \phi_{\ell}\right)^{2}+\frac{1}{2}(\tanh k h-1)\left(k \phi_{\ell}\right)^{2}\right] \mathrm{dS} .
$$

where $W$ is the vertical velocity of the fluid at the free surface due to the horizontal translational oscillations of the body. The value $W$ at $z=0$ can be determined by solving a simple linear problem.

Although the equation (4.16) is very convenient from the computational point of view, however, this quadratic force $\mathrm{F}_{\mathrm{q}}$ can also be determined by the traditional method of fluid dynamics using Green's theorem combined with the application of the radiation condition at infinity. We shall discuss this simple approach to estimating $\mathrm{F}_{\mathrm{q}}$ in a subsequent section.

In addition to the quadratic force $\mathrm{F}_{\mathrm{q}}$ derived from the quadratic part $\phi_{\mathrm{q}}$ of the velocity potential, there is a significant contribution to the second-order force generated by the linear potential, $\phi_{\ell}$. In fact, this additional secondorder force arises from the pressure distributions derived from the potential $\phi_{\ell}$. These pressure distributions have second-order terms which exert an additional wave loading on a structural component. Actually, two forces constitute this additional second-order contribution. One corresponds to the dynamic force, $F_{d}$ which is the resultant of the dynamic pressure, $-\frac{1}{2} p q^{2}\left(q^{2}=\left(\nabla \phi_{l}\right)^{2}\right)$ over the surface of the body. Thus the force $F_{d}$ has the following integral representation

$$
F_{d}=\int_{S} \frac{1}{2} \rho\left(\nabla \phi_{\ell}\right)^{2} d S
$$

where $S$ is the body surface and $n_{x}$ is the direction cosine between the outward normal and the direction of the force $F_{d}$. It should be pointed out that the potential includes the responses of the body to both fluctuating motions and the fluctuating extensional motions due to waves.

Another force provides a second-order contribution to the wave loading on structures whenever the body penetrates the free surface of the liquid. It is directly associated with the transient and hydrostatic pressures of the irrotational flow. According to Lighthill, this additional second-order horizontal $x$-component of the force, $F_{w}$ acting at the waterline $w$ (where the structural 
component intersects the free surface of the fluid) is given by

$$
\mathrm{F}_{\mathrm{w}}=\oint_{\mathrm{w}} \frac{\rho}{2 \mathrm{~g}}\left(\frac{\partial \phi_{\ell}}{\partial t}\right)^{2} \mathrm{dy},
$$

where the integrand is the horizontally resolved force per unit length acting at the waterline and the integral gives its resultant in the $x$-direction.

We denote the linear force $\mathrm{F}_{\ell}$ which is equal to the resultant of the transient pressures $-\left(\rho \frac{\partial \phi_{\ell}}{\partial t}\right)$. This force can therefore be written as

$$
\mathrm{F}_{\ell}=\int_{S}\left(-\rho \frac{\partial \phi}{\partial t}\right) \mathrm{dS} .
$$

This force is generally calculated using purely linear analysis.

It follows from the above discussion that the total second-order contribution to the irrotational-flow loading on an offshore structure is equal to $\mathrm{F}_{\mathrm{d}}+\mathrm{F}_{\mathrm{w}}+\mathrm{F}_{\mathrm{q}}$, whereas the linear wave loading on such structure is $F_{\ell}$. The accuracy of the wave loading can thus greatly be increased by combining $F_{\ell}$ with the nonlinear contribution, $\left(\mathrm{F}_{\mathrm{d}}+\mathrm{F}_{\mathrm{w}}+\mathrm{F}_{\mathrm{q}}\right)$. The latter provides a nonlinear modification of the Morison equation which has a new form

$$
\mathrm{D} \equiv \mathrm{F}_{\ell}+\mathrm{F}_{\mathrm{n} \ell}+\mathrm{F}_{\mathrm{D}} \text {, }
$$

where $\mathrm{F}_{\mathrm{n} \ell}=\mathrm{F}_{\mathrm{d}}+\mathrm{F}_{\mathrm{w}}+\mathrm{F}_{\mathrm{q}}$ is the nonlinear contribution which is made up of the dynamic, waterline, and the quadratic forces associated with the irrotationalflow part of the wave loading on offshore structures. It will be shown in subsequent sections of this paper that the nonlinear contribution to the wave loading on structures is quite significant.

5. LINEAR FORCE AND MORISON'S COEFFICIENT BASED UPON THE LINEAR DIFFRACTION THEORY.

Havelock [1] developed the linear diffraction-theory potential for a long vertical cylinder of radius $b$ in an oncoming wave train in deep water described by the potential given by (4.13a). This potential has the form

$$
\phi_{\ell}=\operatorname{Re}\left[\frac{a \omega}{k}\right] \exp (i \omega t+k z) \sum_{n=0}^{\infty} \alpha_{n} \phi_{n}(k r) \cos \theta,
$$

where Re stands for real part, $\alpha_{0}=1, \alpha_{n}=2(-i)^{n}$ with $n>0$, and 


$$
\phi_{n}(k r)=J_{n}(k r)-\frac{H_{n}^{(2)}(k r) J_{n}^{\prime}(k b)}{H_{n}^{(2) '}(k b)} .
$$

Since our interest only in the $\cos \theta$ term in $\phi_{\ell}$, therefore

$$
\phi_{\ell}=\operatorname{Re}\left[\frac{a \omega}{k} \exp (i \omega t+k z)(-2 i) \phi_{\ell}(k r) \cos \theta\right] .
$$

Thus the linear force, $F_{\ell}(z, t)$ is given by

$$
\begin{aligned}
F_{\ell}(z, t) & =\int_{0}^{2 \pi}\left(-\rho \frac{\partial \phi}{\partial t}\right)_{r=b}(-\cos \theta) b d \theta, \\
& =\operatorname{Re}\left[\frac{2 \rho \pi a b^{2} \omega^{2}}{k b} \exp (i \omega t+k z) \phi_{\ell}(k b)\right], \\
& =C_{M}\left(\rho g a \pi b^{2}\right) \cos (\omega t+\beta),
\end{aligned}
$$

where Morison's coefficient $C_{M}$ and the phase $\beta$ are given by

$$
C_{M} e^{i \beta}=\frac{2}{k b} \phi_{1}(k b)=-\frac{4 i}{\pi(k b)^{2} H_{1}^{(2)^{\prime}(k b)}} .
$$

and the coefficient $C_{M}$ specifies the cylinder's inertial reaction to a fluld acceleration advanced in phase by the phase lead $B$ over its value on the cylinder axis.

In the case of a fluid of finite depth $h$, the corresponding results for $C_{M}$ and $B$ are given by

$$
\begin{aligned}
C_{M} e^{i \beta} & =\frac{2}{k b} \phi_{1}(k b)\left(1-e^{-k h}\right) \tanh k h, \\
& =-\frac{4 i\left(1-e^{-k h}\right) \tanh k h}{\pi(k b)^{2} H_{1}^{(2) '(k b)}} .
\end{aligned}
$$

where the value of $\phi_{1}(\mathrm{~kb})$ is replaced by (5.2) along with the following results

$$
\begin{gathered}
\mathrm{J}_{1}(\mathrm{~kb}) \mathrm{H}_{1}^{(2)^{\prime}}(\mathrm{kb})-\mathrm{H}_{1}^{(2)}(\mathrm{kb}) \mathrm{J}_{1}^{\prime}(\mathrm{kb})=\frac{2}{\pi i k b} \cdot \\
\mathrm{H}_{1}^{(2)}(\mathrm{kb})=\mathrm{J}_{1}(\mathrm{~kb})-i \mathrm{Y}_{1}(k b) .
\end{gathered}
$$

Finally, the values of $C_{M}$ and $\beta$ are explicitly given by 


$$
\begin{gathered}
C_{M}=\frac{4\left(1-e^{-k h}\right) \tanh k h}{\pi(k b)^{2}\left[i J_{1}^{2}(k b)+Y_{1}^{\prime 2}(k b)\right]^{\frac{1}{2}}} \\
B=-\tan ^{-1}\left[\frac{J_{1}^{\prime}(k b)}{Y_{1}^{\prime}(k b)}\right]
\end{gathered}
$$

One of the most significant conclusions of this section is that the Morison coefficient $C_{M}$ decreases or increases according as $k b$ increases or decreases. Physically, when $\mathrm{kb}$ is large (the characteristic dimension $\mathrm{b}$ of the body is large compared with $\mathrm{k}^{-1}$ ), the body becomes efficient as a generator of dipole wave radiation, and the force on it becomes more resistive in nature. This implies that at high frequencies the magnitude of the force increase more slowly than would a purely reactive force.

On the other hand, for small $\mathrm{kb}$ (the characteristic dimension $\mathrm{b}$ of the body is sma11 compared with $\mathrm{k}^{-1}$ ), the body radiates a very sma11 amount of scattered-wave energy. This corresponds to a case of a rigid lid on the ocean inhibiting wave scattering.

6. NONLINEAR WAVE LOADINGS ON A VERTICAL CYLINDER.

We now calculate the linear wave loading, $F_{\ell}$, and the nonlinear wave loading terms $F_{q}, F_{d}$ and $F_{w}$ on a long vertical cylinder of radius $b\left(<<k^{-1}\right)$ which penetrates the free surface of water. With the axis of the cylinder as $x=y=0$, and the presence of the oncoming wave train with potential (4.10), the fluctuating velocity is equal to the value of the horizontal velocity in the undisturbed wave on the cylinder axis. In other words, the fluctuating velocity $U(z, t)$ can be expressed as

$$
U(z, t)=\left(\frac{\partial \phi}{\partial x}\right)_{x=0}=(a \omega k \sin \omega t) \frac{\cosh k(z+h)}{\sinh k h},
$$

To a first approximation, the irrotational response to this fluctuating horizontal velocity produced by the cylinder of radius $b$ is the simple dipole field. The associated velocity potential is given by 


$$
\phi_{\ell}=U(z, t)\left(r+\frac{b^{2}}{r}\right) \cos \theta
$$

in cylindrical polar coordinates $(r, \theta, z)$.

Thus the values of the potential and of the horizontal velocity on the cylinder surface $r=b$ are

$$
\left(\phi_{\ell}\right)_{\mathrm{r}=\mathrm{b}}=2 \mathrm{bU} \cos \theta,\left(\frac{1}{\mathrm{r}} \frac{\partial \phi}{\partial \theta}\right)_{\mathrm{r}=\mathrm{b}}=-2 \mathrm{U} \sin \theta,
$$

In addition to the fluctuating velocity produced near the cylinder by the oncoming waves, waves can also generate significant fluctuating extensional motions as indicated earlier. This extensional motion is the value of the horizontal velocity gradient $\left(\frac{\partial^{2} \phi}{\partial x^{2}}\right)$ in the undisturbed wave train $(4.10)$ on the axis of the cylinder. In other words, the extensional motion is given by

$$
E(z, t)=\left(\frac{\partial^{2} \phi}{\partial x^{2}}\right)_{x=0}=k(-a \omega \cos \omega t) \frac{\cosh k(z+h)}{\sinh k h}
$$

In fluid dynamics, this extension can be treated as a linear combination of a pure dilatation and a dilatationless straining. The local irrotational response to the pure dilatation is the simple monopole field with the velocity potential, $\frac{E}{4}\left(r^{2}-2 b^{2} \log r\right)$. The corresponding response to the dilatationaless straining is a quadrupole field with the potential, $\frac{E}{4}\left(r^{2}+b^{4} r^{-2}\right) \cos 2 \theta$. Thus the combined velocity potential has the form

$$
\phi_{l}=\frac{E}{4}\left(r^{2}-2 b^{2} \log r\right)+\frac{E}{4}\left(r^{2}+\frac{b^{4}}{r^{2}}\right) \cos 2 \theta,
$$

and the horizontal velocity on the cylinder surface is equal to

$$
\left(\frac{1}{r} \frac{\partial \phi}{\partial \phi}\right)_{r=b}=-E b \sin 2 \theta \text {, }
$$

The equations (6.3ab) give a linear force equal to the resultant of the transient pressures $-\left(\rho \frac{\partial \phi_{\ell}}{\partial t}\right)$ so that the linear force per unit height of the cylinder, $\mathrm{F}_{\ell}$ is

$$
F_{\ell}(z, t)=\int_{0}^{2 \pi}\left(-\rho \frac{\partial \phi \ell}{\partial t}\right)(-\cos \theta) b d \theta=2 \rho \pi b^{2} \dot{U},
$$


where the classical Morison coefficient in (6.7) is $C_{M}=2$.

The total linear force, $\overline{\mathrm{F}}_{\ell}$ exerting on the cylinder is given by

$$
\overline{\mathrm{F}}_{\ell}=\int_{-h}^{O} \mathrm{~F}_{\ell}(\mathrm{z}, \mathrm{t}) \mathrm{dz}=2 \pi \rho a g \mathrm{~b}^{2} \tanh k \mathrm{kh} \cos \omega t .
$$

The total drag force, $\overline{\mathrm{F}}_{\mathrm{D}}$ exerting on the cylinder is equal to

$$
\begin{aligned}
\overline{\mathrm{F}}_{\mathrm{D}} & =\int_{-\mathrm{h}}^{0} \frac{1}{2} \rho \mathrm{A} \mathrm{C}_{\mathrm{D}} \mathrm{U}^{2} \mathrm{dz}, \\
& =\frac{1}{2} \rho g \mathrm{~g}^{2} \mathrm{C}_{\mathrm{D}} \tanh \mathrm{kh} \sin ^{2} \omega t+\left[\frac{\sinh 2 \mathrm{kh}}{2 \sinh \mathrm{kh}}-\frac{\mathrm{kh}}{\sinh \mathrm{kh}}\right],
\end{aligned}
$$

Thus the sum of (6.8) and (6.10) gives the total drag D involved in the Morison equation.

We next note that the function $W$ involved in the formula (4.16) for the quadratic force represents the effect of the fluid flows at the free surface generated by the horizontal translational oscillations of the cylinder with unit amplitude and frequency $2 \omega$. This function is also directly proportional to $\cos \theta$ which can also be confirmed by the exact diffraction theory. Therefore, the approximate value of $\mathrm{W}$ near the cylinder for small $\mathrm{kb}$ can be expressed by a simple dipole form

$$
\mathrm{W} \approx(4 \mathrm{k})\left(\frac{\mathrm{b}^{2}}{\mathrm{r}} \cos \theta\right)
$$

where the factor $4 \mathrm{k}$ is extracted from the linearized boundary condition $\phi_{t t}+g \phi_{z}=0$ on $z=0$, for oscillations of frequency $2 \omega$ in order to relate the vertical velocity $W$ at the surface to a local velocity potential given by the second factor in (6.11) so that its radial derivative is $\cos \theta$ on the cylinder surface $r=b$.

In view of the proportionality of $\mathrm{W}$ to $\cos \theta$, the only $\cos \theta$ terms that may be present in $\left(\nabla \phi_{\ell}\right)^{2}$ and $\left(k \phi_{\ell}\right)^{2}$ involved in (4.16) are of interest in this analysis. The term

$$
\left(\nabla \phi_{\ell}\right)^{2}=\left(\frac{\partial \phi_{\ell}}{\partial r}\right)^{2}+\left(\frac{1}{r} \frac{\partial \phi_{\ell}}{\partial \theta}\right)^{2}+\left(\frac{\partial \phi_{\ell}}{\partial z}\right)^{2},
$$

can be computed using the dipole potential (6.2), the combined potential (6.5) 
and the basic potential (4.10). It follows from (6.2) and (6.5) that the square of the horizontal speed is

$$
\begin{aligned}
\left(\frac{\partial \phi_{\ell}}{\partial r}\right)^{2}+\left(\frac{1}{r} \frac{\partial \phi}{\partial \theta}\right)^{2} & =\left[U\left(1-\frac{b^{2}}{r^{2}}\right) \cos \theta+\frac{E}{2}\left(r-\frac{b^{2}}{r}\right)+\frac{E}{2}\left(r-\frac{b^{4}}{r^{3}}\right) \cos 2 \theta\right]^{2} \\
& +\left[U\left(1+\frac{b^{2}}{r^{2}}\right) \sin \theta+E\left(r+\frac{b^{4}}{r^{3}}\right) \sin \theta \cos \theta\right]^{2},
\end{aligned}
$$

The free surface value of this quantity gives a term in $\cos \theta$ in the form

$$
-\frac{1}{2} g(a k)^{2} \sin 2 \omega t \operatorname{coth} k h\left[2 r-\frac{2 b^{2}}{r}+\frac{b^{4}}{r^{3}}+\frac{b^{6}}{r^{5}}\right] \cos \theta .
$$

Similarly, using (6.2) and (4.11), the $\cos \theta$ term in the veritcal speed squared is given by

$$
\left[\left(\frac{\partial \phi_{l}}{\partial z}\right)^{2}\right]_{z=0}=\left\{a \omega \cos \omega t+a \omega k \sin \omega t \cos \theta\left(r+\frac{b^{2}}{r}\right)\right\}^{2} .
$$

The $\cos \theta$ term in this expression is involved only with the cross-term between the two terms in the third bracket of $(6.15)$, and is equal to

$$
-\frac{1}{2} g(a k)^{2} \sin 2 \omega t \tanh k h\left(2 r+\frac{2 b}{r}^{2}\right) \cos \theta
$$

A simple addition of $(6.14)$ and (6.15) gives the $\cos \theta$ term in the total fluid speed squared at the free surface in the form $\frac{1}{2} g(a k)^{2} \sin 2 \omega t \operatorname{coth} k h\left[\left(\frac{4 b^{2}}{r}-\frac{b^{4}}{r^{3}}-\frac{b^{6}}{r^{5}}\right)+\left(2 r+\frac{2 b^{2}}{r}\right)\left(\tanh ^{2} k h-1\right)\right]$,

It turns out from $(4.10)$ and (6.2) that the free surface value of $\left(k \phi_{\ell}\right)^{2}$ is

$$
\left[a \omega \cos \omega t \operatorname{coth} k h+U k\left(r+\frac{b^{2}}{r}\right) \cos \theta\right]^{2} \text {. }
$$

The $\cos \theta$ term involved in this expression is also obtained only from the crossterm between the two terms within the third bracket of $(6.18)$, and has the value

$$
g(a k)^{2}\left(r+\frac{b^{2}}{r}\right) \operatorname{coth} k h \sin 2 \omega t \cos \theta \text {. }
$$

Invoking the results $(6.11),(6.17)$ and (6.19), the quadratic force $F_{q}$ is 
given by

$F_{q}=-\rho \int_{b}^{B} r d r \int_{0}^{2 \pi}(-4 k)\left(\frac{b^{2}}{r} \cos \theta\right)\left[\left(\nabla \phi_{\ell}\right)^{2}+\frac{1}{2}(\tanh k h-1)\left(k \phi_{\ell}\right)^{2}\right] d \theta$,

when $\mathrm{b}<\mathrm{r}<\mathrm{B}$ and the upper limit $\mathrm{B}$ will be determined later on.

Evaluation of $(6.20)$ gives

$$
\begin{aligned}
F_{q} & =2 \pi \rho g k^{3}(a b)^{2} \sin 2 \omega t\left[\left(4 b^{2} \log \frac{B}{b}-\frac{3 b^{2}}{4}+\frac{b^{4}}{2 B^{2}}+\frac{b^{6}}{4 B^{4}}\right)\right. \\
& \left.+\left(B^{2}-b^{2}+2 b^{2} \log \frac{B}{b}\right)\left(\tanh ^{2} k h+\frac{1}{2} \tanh k h-\frac{3}{2}\right)\right] .
\end{aligned}
$$

The overturning moment about the bottom of the cylinder is given by $M_{q}=h F_{q}$.

It may be pointed out that the result $(6.17)$ has an interesting implication for the magnitude of the quadratic force $F_{q}$. In fact, the positive coefficient of the first term in (6.17) clearly indicates that the nonlinear correction to Morison's equation increases with $\mathrm{kb}$. This shows a striking contrast with the result (5.13) based on the linear diffraction theory which predicted that the Morison coefficient $C_{M}$ decreases with $\mathrm{kb}$. In other words, the nonlinear effects become significant in the diffraction regime.

We next estimate the dynamic force, $F_{d}$ which is the resultant of the dynamic pressure, $-\frac{1}{2} p\left(\nabla \phi_{\ell}\right)^{2}$ where $\left(\nabla \phi_{\ell}\right)^{2}$ is to be evaluated on the cylinder surface $r=b$. It follows from (6.13) that the square of the horizontal speed on $r=b$ is

$$
\left[\left(\frac{\partial \phi}{\partial r}\right)^{2}+\left(\frac{1}{r} \frac{\partial \phi}{\partial \theta}\right)^{2}\right]_{r=b}=(2 U \sin \theta+E b \sin 2 \theta)^{2} .
$$

Similarly, the square of the vertical speed on $r=b$ is evaluated from $(6.14)$ and (6.2) to obtain

$\left[\left(\frac{\partial \phi_{l}}{\partial z}\right)\right]_{r=b}=(2 b$ ka $\omega \sin \omega t \cos \theta+a \omega \cos \omega t) \cdot \frac{2 \sinh h^{2} k(z+h)}{\sinh ^{2} k h}$,

so that a simple addition of (6.22) and (6.23) gives the required result for $\left(\nabla \phi_{\ell}\right)^{2}$ on $r=b$ in the form $\left(\nabla \phi_{\ell}\right)^{2}=[2 U \sin \theta+E b \sin 2 \theta]^{2}+$

$+[2 b k a \omega \sin \omega t \cos \theta+a \omega \cos \omega t]^{2} \frac{\sinh ^{2} k(z+h)}{\sinh ^{2} k h}$. 
With this result, the dynamic force per unit lenght of the cylinder is given by

$$
\begin{aligned}
F_{d}(z, t) & =\int_{0}^{2 \pi}\left[-\frac{1}{2} \rho\left(\nabla \phi_{\ell}\right)^{2}\right](-\cos \theta) b d \theta, \\
& =2 \pi \rho b^{2} \operatorname{UE}\left[\frac{1}{2}-\tanh ^{2} k(z+h)\right],
\end{aligned}
$$

where this result is made up of the contributions only from the cross-terms between the terms in the square bracket of the expression (6.24).

The total dynamic force, $\overline{\mathrm{F}}_{\mathrm{d}}$ exerting on the cylinder is given by

$$
\begin{aligned}
\overline{\mathrm{F}}_{\mathrm{d}} & =\int_{-h}^{0} \mathrm{~F}_{\mathrm{d}}(\mathrm{z}, \mathrm{t}) \mathrm{dz}, \\
& =\frac{1}{8} \pi \rho(\mathrm{ab})^{2} \mathrm{gk} \tanh \mathrm{kh}\left[\frac{\sinh 2 \mathrm{kh}-6 \mathrm{kh}}{\sinh ^{2} \mathrm{kh}}\right] \sin 2 \omega t .
\end{aligned}
$$

The overturning moment $M_{d}$ for this case is obtained in the following form

$$
\begin{aligned}
M_{d} & =\int_{-h}^{0}(z+h) F_{d}(z, t) d z \\
& =\frac{\pi \rho(a b \omega)^{2} \sin 2 \omega t}{16 k \sinh k h}\left[(2 k h) \sinh (2 k h)-\cosh (2 k h)+1-6 k^{2} h^{2}\right] .
\end{aligned}
$$

Finally, the magnitude of the waterline force $F_{w}$ is given by

$$
F_{w}=\int_{0}^{2 \pi} \frac{\rho}{2 g}\left(\frac{\partial \phi}{\partial t}\right)(-\cos \theta) b d \theta \text {, }
$$

where

$$
\left[\left(\frac{\partial \phi_{\ell}}{\partial t}\right)^{2}\right]_{z=0}=(2 a b g k \cos \omega t \cos \theta-a g \sin \omega t)^{2} .
$$

Evaluation of the integral (6.30) gives the following result

$$
\mathrm{F}_{\mathrm{w}}=\pi \rho(\mathrm{ak}) \mathrm{ag} \mathrm{b}^{2} \sin 2 \omega t \text {. }
$$

The overturning moment of $\mathrm{F}_{\mathrm{w}}$ about the bottom of the cylinder is simply

$$
M_{\mathrm{w}}=\mathrm{hF}_{\mathrm{w}}=\pi \rho g(\mathrm{ak}) \mathrm{ah} \mathrm{b}^{2} \sin 2 \omega t \text {. }
$$


It is important to point out that in the above calculation, neither the response to the fluctuating velocity by itself nor the response to the fluctuating extension by itself has produced any nonlinear contributions to various wave forces. In fact, all nonlinear contributions come from the interaction of the two kinds of response. In other words, these contributions were found to result only from the cross-terms involving products of the local responses to the fluctuating velocity (6.1) and to the fluctuating extension (6.5).

For the deep water case $(k h \rightarrow \infty)$, the forces $\overline{\mathrm{F}}_{\ell}$ and $\overline{\mathrm{F}}_{\mathrm{d}}$ are calculated from (6.8) and (6.28) in the limit $\mathrm{kh} \rightarrow \infty$ to obtain simpler results due to Lighthill [9].

$$
\begin{aligned}
& \overline{\mathrm{F}}_{\ell}=2 \pi \rho g a b^{2} \cos \omega t, \\
& \overline{\mathrm{F}}_{\mathrm{d}}=\frac{1}{4}(\mathrm{ak}) \pi \rho \mathrm{gab}{ }^{2} \sin 2 \omega t .
\end{aligned}
$$

A simple comparison of these forces reveals that the linear force is independent of the wave number $k$, whereas the dynamic force varies linearly with $k$. This means that the relative importance of the dynamic force increases with the wave steepness ak.

Finally, the total nonlinear contribution to the Morison equation force is equal to the sum of $(6.21),(6.28)$ and $(6.32)$ for the case of fluid of finite depth h.

For the case of deep ocean $(\mathrm{kh} \rightarrow \infty)$, the quadratic force assumes the following limiting form

$$
F_{q}=2 \pi g \rho(a k)(b k)^{2}\left[4 b^{2} \log \frac{B}{b}-\frac{3 b^{2}}{4}+\frac{b^{4}}{2 B^{2}}+\frac{b^{4}}{4 B^{4}}\right] \sin 2 \omega t,
$$

so that the total nonlinear contribution to the total drag is the sum of (6.32), $(6.35)$ and $(6.36)$.

It is interesting to compare these nonlinear contributions in order to determine their relative importance. In the Morison equation limit of small kb, the contribution from $F_{q}$ is relatively small compared with those from $\bar{F}_{d}$ and $F_{w}$ which give 


$$
\left(\overline{\mathrm{F}}_{\mathrm{d}}+\mathrm{F}_{\mathrm{w}}\right)=\left(\frac{1}{2}+2\right)(\mathrm{ak}) g \rho \pi a b^{2} \sin \omega t \cos \omega t .
$$

It follows from this result that the waterline force $F_{w}$ is four times larger than the dynamic force $\overline{\mathrm{F}}_{\mathrm{d}}$. It is also evident that in the 1 imit $\mathrm{kb} \rightarrow 0$, the nonlinear effects are dominated by the sum of the dynamic and waterline forces. On the other hand, the contribution of the quadratic force to the Morison equation increases with increasing value to $\mathrm{kb}$. This means that in the diffraction regime, the nonlinear effects become more important.

For comparison, the Morison equation (1.1) gives a value of the total drag as

$$
D=2 g \rho a \pi b^{2} \cos \omega t+\frac{1}{2} \rho g a^{2} b C_{D} \sin ^{2} \omega t .
$$

The significance fo the nonlinear contribution (6.37) is that it gives an increased positive peak of the total drag force when both $\cos \omega t$ and $\sin \omega t$ are positive.

7. SIMPLE APPROACH TO CALCULATING THE QUADRATIC FORCE.

We assume the following form of the linear potential $\phi_{\ell}$ and the quadratic potential $\phi_{q}$ :

$$
\begin{aligned}
& \phi_{\ell}=\operatorname{Re}\left[\Phi_{\ell} e^{2 i \omega t}\right], \\
& \phi_{q}=\operatorname{Re}\left[\Phi_{q} e^{2 i \omega t}\right] .
\end{aligned}
$$

Inserting these values in equation (4.9), we obtain

$$
\left[\frac{\partial \Phi}{\partial z}-K \Phi_{q}\right]_{z=0}=-\frac{2 i \omega}{g}\left(\nabla \Phi_{\ell}\right)^{2}+\frac{i \omega}{g^{2}} \Phi_{\ell}\left[-\omega^{2} \frac{\partial \Phi \ell}{\partial z}+\frac{\partial^{2} \Phi}{g} \frac{{ }^{2}}{\partial z^{2}}\right]_{z=0} \text {, }
$$

where

$$
K=\frac{4 \omega^{2}}{g} \text {. }
$$

With the potential due to a unit translation oscillation of the body in the form $\operatorname{Re}\left[\Psi \mathrm{e}^{2 i \omega t}\right]$, it follow that on the surface $S$ of the body,

$$
\left[\frac{\partial \psi}{\partial n}\right]_{S}=n_{x} \quad \text { and }\left[\frac{\partial \Phi}{\partial n}\right]_{S}=0
$$

where $n_{x}$ is the $x$-component of a unit inward normal to the surface $s$. 
Furthermore

$$
\left[\frac{\partial \Psi}{\partial n}-K \Psi\right]=0 \text { on } z=0,
$$

where $\Psi$, 1ike $\Psi_{q}$ satisfies the radiation condition at infinity.

This leads us to apply Green's theorem

$$
\iint_{V}\left[\Phi_{q} \nabla^{2} \Psi-\Psi \nabla^{2} \nabla_{q}\right] d V=\int_{S U_{z=0}}\left[\Phi_{q} \frac{\partial \Psi}{\partial n}-\Psi \frac{\partial \Phi}{\partial n}\right] d S,
$$

where the surface integral is taken over the boundaries of the fluid ( $S$ and $z=0$ ) and $\mathrm{n}$ is a normal outward from the fluid.

Since both $\Phi_{q}$ and $\Psi$ satisfy the Laplace equation, the left hand side of (7.6) vainshes so that $(7.6)$ gives

$$
\int_{S \cup z=0}\left[\Phi q \frac{\partial \Psi}{\partial n}-\Psi \frac{\partial \Phi}{\partial n}\right] d S=0
$$

After performing the indicated integration in (7.7) and applying the conditions (7.4ab) and (7.5), it follows from (7.7) that

$$
\int_{z=0} \Psi\left[\frac{\partial \Phi}{\partial z}-K \Phi_{q}\right] d x d y=\int_{S} \Phi_{q} n_{x} d S
$$

Thus the quadratic force $F_{q}$ can be written as

$$
\begin{aligned}
F_{q} & =\int_{S}\left[-\rho \frac{\partial \Phi}{\partial t}\right] n_{x} d S, \\
& =\operatorname{Re}\left[-\rho 2 i \omega e^{2 i \omega t} \int_{S} \Phi_{q} n_{x} d S\right]
\end{aligned}
$$

Invoking (7.8) and (7.3), it follows that

$$
\begin{aligned}
F_{q} & =\operatorname{Re}\left[(-2 i \omega \rho) e^{2 i \omega t} \frac{1}{K} \int_{z=0}\left(\frac{\partial \Psi}{\partial z}\right)_{z=0}\left[\frac{\partial \Phi}{\partial z}-K \Phi_{q}\right] d x d y\right], \\
& =\operatorname{Re}\left[-\frac{4 \rho \omega}{g K} e^{2 i \omega t} \int_{z=0}\left(\frac{\partial \Psi}{\partial z}\right)_{z=0}\left\{\left(\nabla \Phi_{\ell}\right)^{2}-\frac{1}{2 g} \Phi_{\ell}\left(-\omega^{2} \frac{\partial \Phi}{\partial z}+g \frac{\partial^{2} \Phi_{\ell}}{\partial z^{2}}\right)\right\} d x d y\right],
\end{aligned}
$$


$=\operatorname{Re}\left[-\int_{z=0} \mathrm{~W}\left\{\left(\nabla \phi_{\ell}\right)^{2}+\frac{1}{2}(\tanh k h-1)\left(k \phi_{\ell}\right)^{2}\right\} \mathrm{dx} d \mathrm{y}\right]$,

where $W=\left(\frac{\partial \Psi}{\partial z}\right)_{z=0}$ is the vertical velocity on the free surface associated with the unit translational oscillation of the body.

In order to determine the vertical velocity $\mathrm{W}$, it is necessary to find the solution for the potential $\Psi$. As stated earlier, the function $\Psi$ satisfies the Laplace equation, the condition $(7.4 \mathrm{ab})$ on the cylinder $r=b$, the free surface condition on $z=0$ and the radiation condition at infinity. The solution $\Psi$ can be expressed in the following form

$\Psi(\mathrm{r}, \theta, z)=\left[\frac{i k}{\pi}(\mathrm{P}) \int_{-\infty}^{\infty} \frac{\mathrm{K}_{1}(|\alpha| r)}{|\alpha| \mathrm{K}_{1}^{\prime}(|\alpha| \mathrm{b})} \frac{\mathrm{e}^{i \alpha z} \mathrm{~d} \alpha}{\alpha(\mathrm{k}-\mathrm{i} \alpha)}+\frac{2 \mathrm{e}^{\mathrm{Kz}} \mathrm{H}_{1}{ }^{(2)}(\mathrm{Kr})}{\mathrm{KH}_{1}{ }^{(2),}(\mathrm{Kb})}\right] \cos \theta$,

where (P) denotes the Cauchy principle value of the integral and $K_{1}(x)$ is the Bessel function of imaginary argument.

It is noted that the integral in (7.14) represents the standing wave part of the motion and the non-integral part is the radiation component of the motion. The r-derivative of the integral on $\mathrm{r}=\mathrm{b}$ is a simple Fourier integral which can easily be evaluated as $1-e^{2 \mathrm{Kz}}$, and the $\mathrm{r}$-derivative of the radiation component on $\mathrm{r}=\mathrm{b}$ is $2 \mathrm{e}^{\mathrm{Kz}}$ so that the potential $\Psi$ satisfies the boundary condition, $\left(\frac{\partial \Psi}{\partial r}\right)_{r=b}=\cos \theta$.

Some simple algebraic calculation gives the real integral form of $\mathrm{W}$

$$
\mathrm{W}=\left[\frac{2}{\pi} \int_{0}^{\infty}\left\{-\frac{\mathrm{K}_{1}(\mathrm{Kr} \alpha)}{\alpha \mathrm{K}_{1}^{\prime}(\mathrm{Kb} \alpha)}\right\} \frac{\mathrm{d} \alpha}{1+\alpha^{2}}+\frac{2 \mathrm{H}_{1}^{(2)}(\mathrm{Kr})}{\mathrm{H}_{1}^{(2)^{\prime}}(\mathrm{Kb})}\right] \cos \theta \text {. }
$$

From the asymptotic nature of the Bessel function for small $\mathrm{Kr}$ and $\mathrm{Kb}$, it turns out that

$$
\lim _{\substack{\mathrm{Kr} \rightarrow 0 \\ \mathrm{~Kb} \rightarrow 0}}\left[-\frac{\mathrm{K}_{1}(\mathrm{Kra})}{\alpha \mathrm{K}_{1}^{\prime}(\mathrm{Kb} \alpha)}\right]=\frac{\mathrm{Kb}^{2}}{\mathrm{r}} .
$$


Thus the integral in (7.15) is asymptotically equal to $\frac{\mathrm{Kb}^{2}}{\mathrm{r}}$, and the non-integral term is approximately $-\frac{2 \mathrm{~Kb}^{2}}{\mathrm{r}}$ as both $\mathrm{Kr}$ and $\mathrm{Kb}$ tend to zero. This leads to the final asymptotic value of $\mathrm{W}$ as

$$
\mathrm{W} \sim-\frac{\mathrm{Kb}^{2}}{\mathrm{r}} \cos \theta
$$

which is identical with the form (6.10).

In order to estimate the effective range $b<r<B$ within which (7.17) holds, we can define $B$ by the equation

$$
\operatorname{Re}\left[\int_{b}^{\infty} \mathrm{W} d r\right]=-\mathrm{Kb}^{2} \log \left(\frac{\mathrm{B}}{\mathrm{b}}\right) \cos \theta
$$

On the other hand, it follows from (7.15) that

$$
\int_{b}^{\infty} \mathrm{W} d r=\left[\frac{2}{\pi} \int_{0}^{\infty}\left\{-\frac{\mathrm{K}_{\mathrm{o}}(\mathrm{Kb} \alpha)}{\alpha^{2} \mathrm{~K} \mathrm{~K} \mathrm{~K}_{1}^{\prime}(\mathrm{Kb} \alpha)}\right\} \frac{\mathrm{d} \alpha}{1+\alpha^{2}}+\frac{2 \mathrm{H}_{\mathrm{o}}^{(2)}(\mathrm{Kb})}{\mathrm{KH}_{1}^{(2)^{\prime}(\mathrm{Kb})}}\right] \cos \theta \text {. }
$$

We next use the asymptotic result for small $\mathrm{Kb}$ as

$$
\left[-\frac{\mathrm{K}_{\mathrm{O}}(\mathrm{Kb} \alpha)}{\mathrm{K} \alpha^{2} \mathrm{~K}_{1}^{\prime}(\mathrm{Kb} \alpha)}\right] \sim-\mathrm{Kb}^{2}\left[\log \left(\frac{1}{2} \mathrm{~Kb} \alpha\right)+\gamma\right]
$$

to obtain the asymptotic representation for (7.19) in the form

$$
\int_{b}^{\infty} \mathrm{W} d r \sim\left[\mathrm{Kb}^{2}\left\{\log \left(\frac{\mathrm{Kb}}{2}\right)+\gamma\right\}+2 \mathrm{~Kb}^{2}\left\{\log \left(\frac{\mathrm{Kb}}{2}\right)+\gamma+\frac{i \pi}{2}\right\}\right] \cos \theta
$$

where $\gamma$ is Euler's constant.

This result agrees with (7.18) provided that

$$
B=\frac{1}{K}\left(2 e^{-\gamma}\right)=\left(\frac{1}{2} e^{-\gamma}\right)(k \tanh k h)^{-1} \equiv C(k \tanh k h)^{-1} \text {, }
$$

where $C \equiv \frac{1}{2} e^{-\gamma}$ is a numerical constant.

It is noted here that beyond the limiting radius $r=B, W$ is oscillatory and hence makes a small contribution to the quadratic force $\mathrm{F}_{\mathrm{q}}$ discussed in the previous section. 


\section{CONCLUDING REMARKS.}

This work has been devoted to a nonlinear modification of the classical Morison equation. It has been shown that the nonlinear contribution to the Morison equation is $\left(\mathrm{F}_{\mathrm{d}}+\mathrm{F}_{\mathrm{w}}+\mathrm{F}_{\mathrm{q}}\right)$ which is the sum of the dynamic, waterline, and the quadratic wave forces associated with the irrotational component of the fluid flows. The salient feature of the nonlinear theory has been illustrated by means of a large vertical cylinder immersed in an ocean.

With regard to the geometrical shape of offshore structures, it can be added here that the present theory is general enough to incorporate any other axisymmetric solid structures of practical interest.

ACKNOWLEDGEMENT. Authors express their grateful thanks to Professor Sir James Lighthill for his continued interest and helpful suggestions during the preparation of this paper. This work was supported by the National Research Council of Canada, and by East Carolina University.

\section{REFERENCES}

1. Havelock, T.H., The pressure of water waves on a fixed obstacle, Proc. Roy. Soc. Al75 (1940) pp. 409-421.

2. Morse, P.M., Vibration and Sound. McGraw Hill Book Company, Inc., New York, 2953 , p. 346.

3. MacCamy, R.C., and Fuchs, R.A., Wave Forces on Piles, A Diffraction theory. Beach Erosion Board Technical Memo. No 69 (1954).

4. Chakraborty, S.K., Nonlinear Wave Forces on Vertical Cylinder, Journal of the Hydraulics Division, ASCE. Vo1. 98, No. HY 11, Proc. Paper 9333, (1972) pp. 1895-1909.

5. Chakraborty, S.K., Second-order Wave Forces on Large Vertical Cylinder, Jour. Waterways Harbors and Coastal Engg. Division, ASCE 101, No. WW3, (1975) pp. 311-317.

6. Raman, H. and Venkatanarasaiah, P., Forces due to Nonlinear Waves on Vertical Cylinders, Proc. ASCE 102, No. WW3 (1976) pp. 301-316.

7. Morison, J.R., O'Brien, M.P., Johnson, J.W., and Schaaf, S.A., The Forces exerted by Surface Waves on Piles, Petroleum Transactions, AIME Vo1. 189 (1950) pp. 149-154.

8. Hogben, N., Miller, B.L., Searle, J.W., and Ward, G., Estimation of Fluid Loading on Offshore Structures" National Maritime Institute Report No. 11 (1977) pp. 1-60. 
9. Lighthill, M.J. Waves and Hydrodynamic Loading, Proc. Second. International Conference on the Behaviour of Off-shore Structures. Vo1. 1 (1979) pp. 1-40.

Present Address of the authors:

Dr. Lokenath Debnath

Mathematics Department

East Carolina University

Greenville, North Carolina 27834, U.S.A.

Dr. Matiur Rahman

Applied Mathematics Department

Technical University of Nova Scotia

Halifax, Nova Scotia, Canada. 


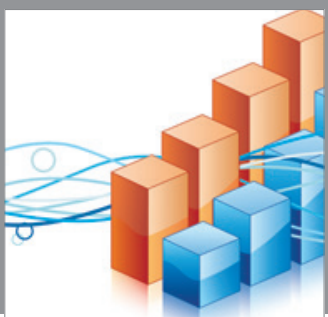

Advances in

Operations Research

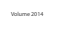

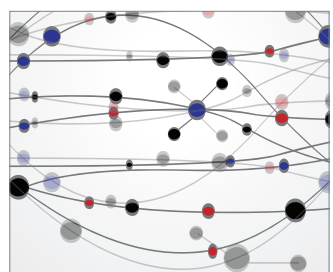

\section{The Scientific} World Journal
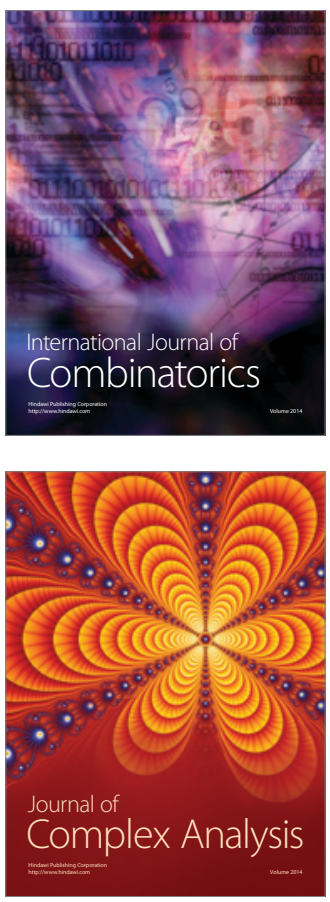

International Journal of

Mathematics and

Mathematical

Sciences
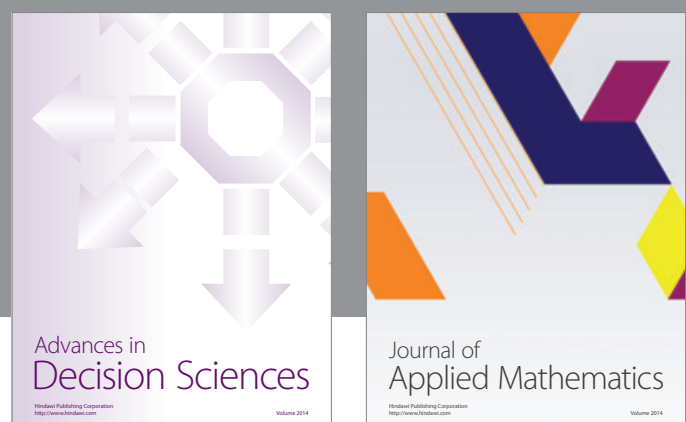

Journal of

Applied Mathematics
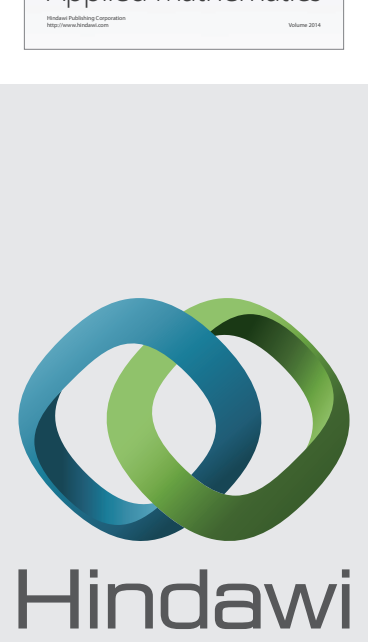

Submit your manuscripts at http://www.hindawi.com
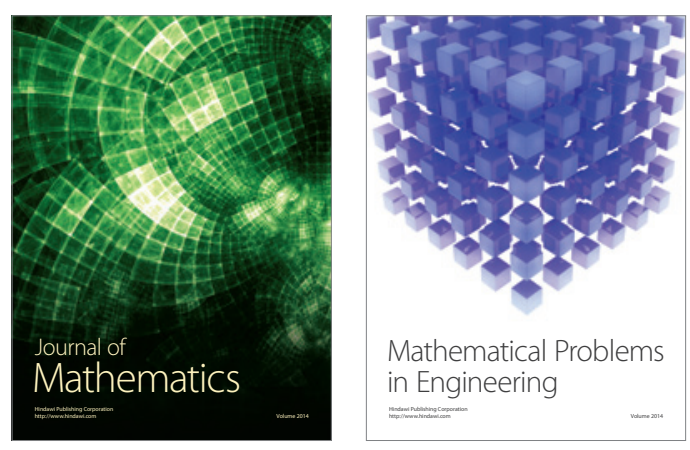

Mathematical Problems in Engineering
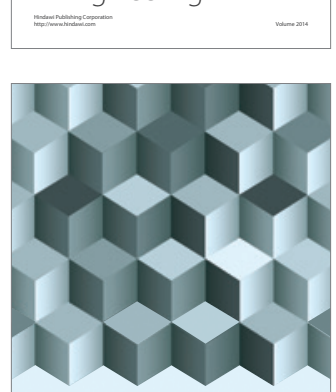

Journal of

Function Spaces
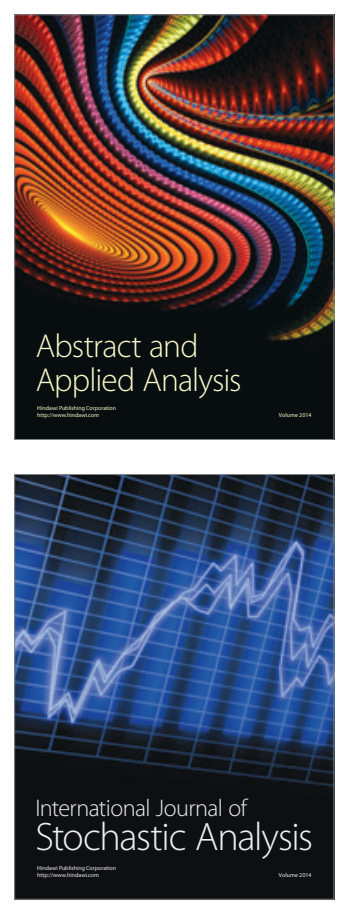

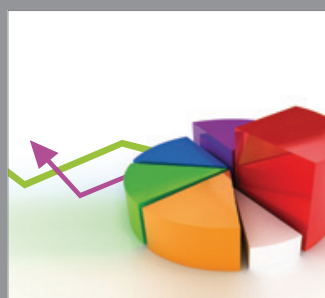

ournal of

Probability and Statistics

Promensencen
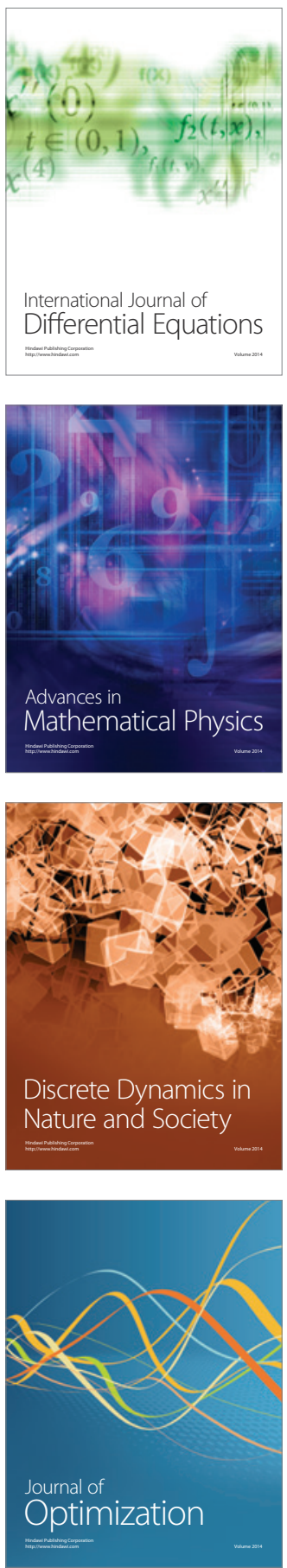